\title{
Verhaltenssüchte bilden eine eigene diagnostische Kategorie
}

\author{
Behavioural Addictions are a Separate Diagnostic Entity
}

Bibliografie

DOI $10.1055 / \mathrm{s}-2007-986308$

Psychiat Prax 2008; 35:

$160-162$

(c) Georg Thieme Verlag KG

Stuttgart · New York .

ISSN 0303-4259

\section{Korrespondenzadressen}

Prof. Dr. Jobst Böning

Klinik und Poliklinik für

Psychiatrie und Psychotherapie

der Universität Würzburg

Füchsleinstraße 15

97080 Würzburg

boening@mail.uni-

wuerzburg.de

\section{Dr. Hans Watzl}

Universität Konstanz, FB Psychologie

78457 Konstanz

hans.watzl@uni-konstanz.de

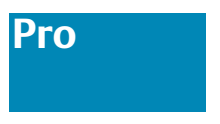

Bei der Weiterentwicklung von Standards zur Operationalisierung psychischer Störungen und einhergehender Probleme der Klassifikation und differenziellen Diagnostik wird in jüngster Zeit wieder verstärkt die Parallelität zwischen stoffgebundenen und stoffungebundenen Suchterkrankungen diskutiert. Dabei werden unter den stoffungebundenen „Tätigkeitssüchten“ (im Folgenden „Verhaltenssüchte“ genannt) exzessive belohnungssuchende und autonom gewordene Verhaltensweisen (z. B. Glücksspiele, Internetnutzung) verstanden, bei denen die Betroffenen die diagnostischen Kriterien einer Abhängigkeitserkrankung erfüllen. Die auf unterschiedlichen Untersuchungs- und Funktionsebenen gewonnenen Forschungsergebnisse v.a. aus der Lerntheorie und Neurobiologie legen nahe, dass im Rahmen eines biopsychosozialen Modells zur Entstehung und Aufrechterhaltung süchtigen Verhaltens gleichermaßen stoffgebundene wie stoffungebundene Abhängigkeitserkrankungen in denselben zentralnervösen Mechanismen verankert sind [1].

Bislang fanden „Verhaltenssüchte“ noch keinen Eingang als eigenständiges Störungsbild in die gängigen Klassifikationssysteme psychischer Störungen [2]. Derzeit kann eine „Verhaltenssucht" nur in Anlehnung an die Einordnung des „Pathologischen Spielens“ als „Störung der Impulskontrolle, nicht andernorts klassifiziert" diagnostiziert werden. Diese Diagnose ist jedoch im Hinblick auf psychopathologisch-phänomenologische, neurobiologische, differenzialdiagnostische, präventive und therapeutische Implikationen völlig unzureichend.

Einige Autoren fassen pathologisches Glücksspiel als sog. „Zwangsspektrumsstörung“ auf [3], wobei in diesem Erklärungsmodell aber zwischen initial ich-syntonem verstärkenden Belohnungsverhalten und finalem ich-dystonem „zwanghaften Sich-so-verhalten-Müssen“ zu differenzieren wäre. Offenbar kann letzteres inzwischen „impulskontrollgestört-zwanghaftes“ Verhalten durch Aktivierung derselben orbitofronto-striato-thalamischen Regelkreise in Gang gesetzt werden, die auch an der Entstehung von „primären“ Zwangssymptomen beteiligt sind (vgl. in [1]).

Andere Autoren postulieren inzwischen wieder, dass das Erscheinungsbild der krankhaft exzessiv durchgeführten Verhaltensweisen mit den Merkmalen von Abhängigkeitserkrankungen vergleichbar ist und formulieren z. B. den Begriff der „Glücksspielsucht“ als Prototyp einer Verhaltenssucht [4-6]. Als zentrale Bestimmungsmerkmale gelten dabei ein erlernter Kontrollverlust über das Verhalten (inkl. Toleranzentwicklung) und die Vernachlässigung anderer wichtiger Lebensbereiche sowie psychovegetative Entzugserscheinungen. So zeigt sich ein dringender Handlungsbedarf, um für bestimmte in der Praxis auftretende pathologische exzessive Verhaltensweisen eine Operationalisierung im Sinne der diagnostischen Kategorienbildung zu etablieren.

Diese Auseinandersetzung wird derzeit auch im Wissen um die kommende 11. Revision der „Internationalen Krankheitsklassifikation“ (ICD-11) und der 5. Revision des „Diagnostic and Statistical Manuals“ (DSM-V) geführt. Die dazu notwendigen wissenschaftlichen Vorarbeiten von Weltgesundheitsorganisation (WHO) und American Medical Association (AMA) haben bereits begonnen. Zwar erfolgte unlängst eine vorläufige $A b-$ lehnung einer eigenen Diagnosekategorie einer Form der Verhaltenssucht z.B. „Video game addiction“ im DSM-V durch die AMA. Der wissenschaftliche Beirat unterstreicht in seinem Report zur Computerspielsucht (www.ama-assn.org) aber deutlich den Handlungsbedarf, merkt den Mangel an empirischen Studien an und schlägt diese Kategorie immerhin als Forschungsdiagnose vor. Die Revisionen der Klassifikationssysteme psychischer Störungen orientieren sich an einem 
studienbasierten Vorgehen im Sinne der „Evidence-Based Medicine“, womit sichergestellt werden soll, dass ein methodisch begründeter Erkenntnisgewinn (basierend auf wissenschaftlicher Methodik, kontrollierten klinischen Studien und reproduzierbaren Ergebnissen, vgl. [7]) in den Modifikationen dieser Systeme implementiert wird.

Nach den bisherigen Forschungsergebnissen zu den exzessiv durchgeführten Verhaltensweisen mit pathologischem Charakter $[6,8]$ zeigt sich, dass häufig die Kriterien der Substanzabhängigkeiten zur Operationalisierung der Symptome genutzt werden, um die Symptomkomplexe empirisch bzw. phänomenologisch trennscharf zu erfassen. Obwohl bei den derart zusammengefassten Störungsbildern dem Organismus keine psychotrop wirksamen Substanzen von außen zugeführt werden, treten viele Symptome mit nahezu vollständiger Übereinstimmung im Vergleich zu den stoffgebundenen Abhängigkeitserkrankungen auf. Für die Aufrechterhaltung eines Verhaltens scheinen körpereigene biochemische Veränderungen und neuronale Konditionierungs- und Bahnungsprozesse, die durch die exzessiven belohnungssuchenden Verhaltensweisen ausgelöst werden, verantwortlich zu sein. Verschiedene Studienergebnisse zeigen ebenso, dass der pathologischen exzessiven Verhaltensausübung und der Substanzabhängigkeit vergleichbare neurobiologische Mechanismen zugrunde liegen, wie beispielsweise bei pathologischen Glücksspielern eine veränderte Aktivierung der Basalganglien und des frontalen und orbitofrontalen Kortex (z.B. [6]). Gleichfalls wies man bei Betroffenen von „Verhaltenssüchten" bereits analoge psychophysiologisch messbare Muster nach, wie sie bei Alkohol oder Cannabis bekannt sind (z. B. [9]). So scheint vergleichbar zur Substanzabhängigkeit die Funktion der Verhaltenssucht in einer (dysfunktional) erlernten Regulationsmöglichkeit des Gehirns zu liegen, die den Betroffenen ermöglicht, ihre „Biochemie der Gefühle“ wieder ins Gleichgewicht zu bringen und dabei effektiv Stress zu reduzieren.

Oft wird das parallele Auftreten von Symptomen des pathologischen exzessiven Verhaltens in Verbindung mit anderen psychiatrischen Erkrankungen (z.B. Depression, Angststörungen) dahingehend kritisch gewertet, dass „Verhaltenssüchte“ nur eine Begleiterscheinung von einer anderen als Primärdiagnose gestellten psychiatrischen Erkrankung seien [10]. Betrachtet man in diesem Zusammenhang die Verhältnisse bei den stoffgebundenen Störungen, so fällt auf, dass auch diese Patienten eine hohe Komorbiditätsrate vornehmlich aus dem Spektrum der neurotischen und Persönlichkeitsstörungen aufweisen. Die Diagnose der Substanzabhängigkeit führt hier jedoch im Regelfall dazu, dass das klinische Gesamtbild des Patienten durch die Vergabe mehrerer Diagnosen detailreicher beschrieben wird, was letztlich zur Identifizierung der Grundkonflikte und Optimierung des Heilerfolges beiträgt. In diesem Zusammenhang wird die Aufgabe der Diagnostik im Sinne einer differenzierten und verhaltensnahen Beschreibung des gesamten Störungsbildes auch unter Einbeziehung der pathologisch exzessiven Verhaltensweisen - augenfällig. Die genaue Charakterisierung der Symptome und klare diagnostische Kriterien sollten in der Praxis das gegenwärtige Defizit bei der Diagnostik von „Verhaltenssüchten“ beheben. Schließlich muss eine zukünftig auch ätiologieorientierte psychiatrische Diagnostik in ihrem Ergebnis zu einer möglichst detailreichen Beschreibung der pathogenen psychischen Anteile des Patienten führen.

\section{Kontra}

Die Diskussion, ob der Begriff „Sucht“ für die Klassifikation psychischer Störungen sinnvoll ist und ob er auf mehr Störungen anzuwenden ist als auf die Abhängigkeit von Alkohol, Barbituraten, Opiaten u.ä., ist so alt wie die psychiatrische Krankheitslehre. Bereits 1905 [11] publizierte der Würzburger Ordinarius Rieger den Aufsatz „Über die Trunksucht und die ,Suchten“ überhaupt“. Rieger unterscheidet darin drei Suchtformen, die sich jeweils in zahlreiche Unterformen aufgliedern: 1. die „moralischen Süchte“, zu denen die Spielsucht gehört, 2. die „pathologischen Süchte“ mit Krankheiten wie Gelbsucht, und 3. die „Kontaminationen“ aus den beiden ersten, z.B. Trunksucht, Eifersucht, Verschwendungssucht. Eine Einteilung, die sich nicht an der Stoffgebundenheit orientierte!

Die Frage der Eingrenzung oder Ausweitung des Suchtbegriffs beherrschte bei einigen Gelegenheiten die Diskussion in Fachzeitschriften. Victor von Gebsattel gab einer Publikation den Titel „Süchtiges Verhalten im Gebiet sexueller Verirrungen“ [12]. Dies griff Giese (z. B. [13]) wieder auf, um „sexuell-süchtiges Verhalten von einer bloßen Devianz oder einem sexuellen Fehlverhalten“ abzugrenzen. Er sah folgende Analogien zu den „stoffgebundenen“ Süchten, fast identisch zu den heute diskutierten: „... die zunehmende Zentrierung der Denk- und Vorstellungsräume in Richtung auf den angestrebten Erlebniszustand, die zunehmende Frequenz der zugehörigen Handlungsvollzüge bei abnehmender Triebbefriedigung, die dranghafte Unruhe und Unerwehrbarkeit in Richtung der Durchführung, das Auftreten körperlich-vegetativer Symptome bei Ausbleiben oder Verhinderung“ [14]. Bemerkenswerterweise scheint diese Diskussion um Paraphilien und ähnliche Verhaltensweisen in Vergessenheit geraten zu sein.

Beim nächsten Anlass - Mitte der 80er-Jahre - wurde die Klassifikation pathologischen Spielverhaltens an Spielautomaten oder in Spielcasinos diskutiert. Während einige Autoren bei exzessiven Glücksspielern „alle Symptome einer psychischen Abhängigkeit“ sahen und ein Krankheitsbild der „Glücksspielsucht“ beschrieben $[15,16]$, wurde dies von anderen als „mystisch-biologisches Suchtmodell“ [17] abgelehnt.

Nach unserem Eindruck kommen in dieser Diskussion zwei Grundpositionen zum Ausdruck. Die Vertreter der „Verhaltenssüchte“ gehörten oft zur psychodynamischen oder phänomenologischen Richtung der Psychopathologie. Sie betonen bei diesen „nicht stoffgebundenen Süchten“ eine phasenhafte, irreversible Abhängigkeitsentwicklung, die in Kontrollverlust endet. Auf der anderen Seite finden sich Vertreter eines möglichst klaren und eindeutigen Klassifikationssystems, oft Verhaltenstherapeuten, aber auch psychiatriekritische Gegner einer „Medizinisierung“ devianten Verhaltens. Zum Teil betonen diese eher die Ähnlichkeiten mit Impulskontrollstörungen (wie Trichotillomanie oder Pyromanie) oder mit den Zwangsstörungen.

Das einzige neue Argument, das - diesmal von den Vertretern der Verhaltenssuchtposition - angeführt wird, ist das der „Parallelität zwischen zugrunde liegenden psychophysiologischen Mechanismen bei der „Substanzabhängigkeit“ und z. B. dem pathologischen Glücksspiel [18]. Aber differenzieren die ähnlichen Aktivierungsmuster in striatalen und präfrontalen Bereichen bei Darbietung relevanter Reize beide Störungen von anderen $\mathrm{Zu}$ standsbildern, in denen bestimmte Umweltreize Signale für Annäherungsverhalten sind? Hier ist doch nach der Spezifität solcher Befunde zu fragen. Auch fehlt der Nachweis der bei beiden Störungen gleichartigen Kovariation solcher Auffälligkeiten mit klinisch wichtigen Abstufungen beider Störungen. Nach unserer 
Meinung werden auf absehbare Zeit diese psychophysiologischen Mechanismen keinerlei spezifische diagnostische, geschweige denn therapeutische Hilfen bieten können.

Damit bleiben die klassischen Kriterien für die Definition von Krankheitseinheiten: Phänomenologie und Symptombild (Verhalten und Erleben), Verlauf, Behandlung. Die Unterschiede zwischen einer Alkoholabhängigkeit und einer „Kaufsucht“ erscheinen uns dabei eklatant. Die zentralen Punkte bilden dabei die unmittelbaren Effekte des Alkohols und die direkten körperlichen Folgen lang anhaltenden exzessiven Alkoholkonsums. Weder führt die „Kaufsucht“ bei ihrer Ausübung zu vergleichbaren Effekten auf Stimmung, kognitive Fähigkeiten und Bewusstsein, noch hat langjährige „Kaufsucht“ ähnliche organische, zentralnervöse und psychische Veränderungen zur Folge. Zweifellos gibt es daneben auch Gemeinsamkeiten in Erleben, Verlauf und sozialen Folgen, wie sie im o.a. Zitat von Giese (1962) deutlich werden. Solche Gemeinsamkeiten bestehen allerdings auch zwischen „Kaufsucht“ und Zwangsverhalten.

Auf zwei weitere Argumente von Grüsser et al. [18] soll hier wenigstens kurz eingegangen werden:

1. Es „lassen sich klare ... Kriterien für die Diagnostik einer Verhaltenssucht formulieren“. Zweifellos finden sich in der Literatur eindrucksvolle Fallbeispiele. Wir halten dennoch die dort in Tab. 1 aufgeführten Kriterien für kaum geeignet, anderes nicht suchthaftes, sehr häufiges und selbstschädigendes Verhalten davon abzugrenzen. Zum einen ergeben sich dabei ähnliche definitorische Probleme wie bei der wenig reliablen Diagnose des „Substanzmissbrauchs“, der kaum von einem als „kritisch“ angesehenen Konsummuster zu unterscheiden ist. Zum anderen beziehen sich diese Kriterien ausschließlich auf subjektives Erleben, d. h. Selbstberichten der Betroffenen, was für forensische Fragestellungen problematisch sein kann. Auf „die Gefahr eines inflationären Gebrauchs des Begriffes“ weisen Grüsser et al. [18] selbst hin.

2. „Die Einordnung... der Verhaltenssucht unter die Störungen der Impulskontrolle ... kann verhindern, dass geeignete Behandlungsmaßnahmen aus dem Bereich suchtkranker Patienten angewendet werden." Betrachtet man aber die in der Literatur beschrieben Therapieverfahren für exzessives Glücksspiel oder Einkaufen, so wird meist die diagnostische Klassifikation für therapeutisch nicht entscheidend gehalten [19] oder sie orientieren sich explizit nicht an einem Suchtmodell $[20,21]$.

Die Frage, ob hochfrequentes und selbstschädigendes Verhalten in diagnostischer, ätiologischer und therapeutischer Hinsicht den stoffgebundenen Abhängigkeiten entspricht, ist wichtig für die Nosologie psychischer Störungen. Aber nicht, weil durch eine solche Gleichsetzung die Krankheitslehre vereinfacht würde, sondern weil sie uns anhält, Konstrukte wie Abhängigkeit, Verlangen oder Kontrollverlust präzise zu operationalisieren und auf ihren pathognomonischen Wert zu prüfen.

\section{Literatur}

1 Böning J. Allgemeine und spezielle Modellvorstellungen zur Sucht. In: Riederer P, Laux G (Hrsg): Neuro-Psychopharmaka (Bd. 6, 2. Aufl.). Wien: Springer, 2005: 209-233

2 Saß H, Wittchen HU, Zaudig M, Houben I. Diagnostische Kriterien des Diagnostischen und Statistischen Manuals Psychischer Störungen DSM-IV-TR. Göttingen: Hogrefe, 2003

3 Hand I. Negative und positive Verstärkung bei pathologischen Glücksspielen: Ihre mögliche Bedeutung für die Theorie und Therapie von Zwangsspektrumsstörungen. In: Hand I (Hrsg): Impulskontrollstörungen - Nichtstoffgebundene Abhängigkeiten - Zwangsspektrumsstörungen. Verhaltenstherapie 2004; 14: 133 - 144

4 Grüsser SM, Poppelreuter S, Heinz A, Albrecht U, Saß H. Verhaltenssucht: Eine eigenständige diagnostische Einheit? Nervenarzt 2007; 78: $997-1002$

5 Petry NM. Should the scope of addictive behaviors be broadened to include pathological gambling? Addiction 2006; Suppl 1: 152 - 160

6 Potenza MN. Should addictive disorders include non-substance-related conditions? Addiction 2006; Suppl 1: 142-151

7 Giere W. Klassifikation in der Medizin. Bundesgesundheitsblatt - Gesundheitsforschung. Gesundheitsschutz 2007; 50: 913-923

8 Lejoyeux N, Mc Loughlin M, Adès J. Epidemiology of behavioral dependence: literature review and results of original studies. Eur Psychiatry 2000; $15:$ 129-134

9 Thalemann R, Wölfling K, Güsser SM. Specific cue-reactivity on computer game related cues in excessive gamers. Behav Neurosci 2007; 121: $614-618$

10 Kratzer S, Hegerl $U$. Ist „Internetsucht“ eine eigenständige Erkrankung? Eine Untersuchung von Menschen mit exzessiver Internetnutzung. Psychiat Prax 2007; Jun 1 [Epub ahead of print] DOI: 10.1055/s2007-970888

11 Rieger K. Über die Trunksucht und die „Suchten“ überhaupt. Festschrift zur Feier des fünfzigjährigen Bestehens der Unterfränkischen Heil- und Pflegeanstalt Werneck. Jena: Fischer, 1905

12 Gebsattel VE von. Süchtiges Verhalten im Gebiet sexueller Verirrungen. Monatsschrift für Psychologie und Neurologie 1932; 82: 113 - 177

13 Giese H. Abnormes und perverses Verhalten. In: Giese H (Hrsg): Psychopathologie der Sexualität. Stuttgart: Enke, 1962: 305-470

14 Schumacher $W$. Untersuchungen zur Psychodynamik des abhängigen Spielverhaltens. In: Feuerlein W (Hrsg): Theorie der Sucht. Heidelberg: Springer, 1986: 165 - 179

15 Kellermann B. Pathologisches Glücksspielen und Suchtkrankheit. Suchtgefahren 1987; 33: 110-120

16 Meyer G. Die stimulierende Wirkung des Glücksspiels. Suchtgefahren 1987; 33: $102-109$

17 Klepsch R, Hand I, Wlazlo Z, Kaunisto E, Friedrich B. Langzeiteffekte multimodaler Verhaltenstherapie bei krankhaftem Glücksspiel. I. Retrospektive Katamnese der Hamburger Pilot-Studie. Suchtgefahren 1987; 33: $137-147$

18 Grüsser SM, Poppelreuter S, Heinz A, Albrecht U, Saß H. Verhaltenssucht: Eine eigenständige diagnostische Einheit? Nervenarzt, 2007 (im Druck)

19 Petry J. Glücksspielsucht: Entstehung, Diagnostik und Behandlung. Göttingen: Hogrefe, 2003

20 Hand I. Negative und positive Verstärkung bei pathologischem Glücksspielen: Ihre mögliche Bedeutung für die Theorie und Therapie von Zwangsspektrumstörungen. Verhaltenstherapie 2004; 14: 133 144

21 Müller A, Mitchell JE, Mertens C, Müller U, Silbermann A, Burgard M, de Zwaan $M$. Comparison of treatment seeking compulsive buyers in Germany and the United States. Behaviour Research and Therapy 2007; 45: $1629-1638$ 